Review Article

\title{
Bottlenecks in the Efficient Use of Advanced Therapy Medicinal Products Based on Mesenchymal Stromal Cells
}

\author{
Natalia Escacena, ${ }^{1}$ Elena Quesada-Hernández, ${ }^{2}$ Vivian Capilla-Gonzalez, ${ }^{1}$ \\ Bernat Soria, ${ }^{1,3}$ and Abdelkrim Hmadcha ${ }^{1,3}$ \\ ${ }^{1}$ Department of Stem Cells, Andalusian Centre for Molecular Biology and Regenerative Medicine (CABIMER), \\ 41092 Seville, Spain \\ ${ }^{2}$ Newbiotechnic (NBT), Bollullos de la Mitación, 41110 Seville, Spain \\ ${ }^{3}$ CIBER de Diabetes y Enfermedades Metabólicas Asociadas (CIBERDEM), 08036 Barcelona, Spain
}

Correspondence should be addressed to Bernat Soria; bernat.soria@cabimer.es

and Abdelkrim Hmadcha; karim.hmadcha@cabimer.es

Received 24 July 2014; Accepted 5 March 2015

Academic Editor: Claudia Montero-Menei

Copyright (C) 2015 Natalia Escacena et al. This is an open access article distributed under the Creative Commons Attribution License, which permits unrestricted use, distribution, and reproduction in any medium, provided the original work is properly cited.

\begin{abstract}
Mesenchymal stromal cells (MSCs) have been established as promising candidate sources of universal donor cells for cell therapy due to their contributions to tissue and organ homeostasis, repair, and support by self-renewal and multidifferentiation, as well as by their anti-inflammatory, antiproliferative, immunomodulatory, trophic, and proangiogenic properties. Various diseases have been treated by MSCs in animal models. Additionally, hundreds of clinical trials related to the potential benefits of MSCs are in progress. However, although all MSCs are considered suitable to exert these functions, dissimilarities have been found among MSCs derived from different tissues. The same levels of efficacy and desired outcomes have not always been achieved in the diverse studies that have been performed thus far. Moreover, autologous MSCs can be affected by the disease status of patients, compromising their use. Therefore, collecting information regarding the characteristics of MSCs obtained from different sources and the influence of the host (patient) medical conditions on MSCs is important for assuring the safety and efficacy of cell-based therapies. This review provides relevant information regarding factors to consider for the clinical application of MSCs.
\end{abstract}

\section{Introduction}

MSCs are considered a heterogeneous population of nonhaematopoietic progenitor cells derived from the mesodermal germ layer that have both self-renewal and multidifferentiation [1] abilities. MSCs found in virtually all postnatal organs and tissues [2] possess multifaceted features, making them promising candidate sources of donor cells for use in cell therapy and transplantation. MSCs function in the repair and support of tissues, contributing to tissue homeostasis. Although the exact origin of MSCs remains elusive, strong evidence has indicated that MSC progenitors are in the perivascular zone [3] in an environment that promotes a quiescent-resting state, ensuring homeostasis maintenance. When a tissue is damaged and the whole machinery of the organism begins to operate the body's repair mechanisms,
MSCs enter the blood stream and are attracted by proinflammatory cytokines at injury areas. Thus, MSCs have been called "guardians of inflammation" [4]. The cytoskeleton, extracellular matrix molecules, cell contacts, adhesion ligands, and receptors are involved in the repair process [5]. Although the exact mechanisms related to the migration of MSCs into specific sites and across the endothelial cell layer remain unknown, chemokines and their receptors may play roles in this process.

Although MSC survival, permanent engraftment, and differentiation into resident cells was thought to be necessary to obtain the beneficial effects of these cells initially, clinical experience and several experiments have shown that one of the primary functions of MSCs, most likely their key function, is to secrete several bioactive molecules related to the microenvironment in which these cells are immersed. 
TABLE 1: Summary of mesenchymal stroma cell nomenclature.

\begin{tabular}{lcc}
\hline Nomenclature & Year & Authors' references \\
\hline CFU-F and osteogenic stem cells & 1974 & Friedenstein et al. [10] \\
Stromal stem cells & 1988 & Owen and Friedenstein [16] \\
Mesenchymal stem cells & 1991 & Caplan [17] \\
Mesenchymal progenitor cells & 1999 & Dennis et al. [18] \\
Skeletal stem cells & 2000 & Bianco and Robey [19] \\
MAPCs and mesodermal progenitor cells & 2002 & Jiang et al. [20] \\
Multipotent mesenchymal stromal cells & 2006 & Dominici et al. (ISCT) [21] \\
Medicinal signalling cells & 2010 & Caplan [22] \\
\hline
\end{tabular}

CFU-F: colony-forming unit fibroblasts; MAPCs: multipotent adult progenitor cells; ISCT: International Society for Cell Therapy.

MSCs secrete a wide variety of proinflammatory and antiinflammatory cytokines, chemokines, growth factors, and prostaglandins under resting and inflammatory conditions [6]. These molecules are associated with immunomodulation (indoleamine-2,3-dioxygenase (IDO), prostaglandin-E2 (PGE-2), TGF- $\beta$, HLA-G5, and HGF), antiapoptosis (VEGF, GM-CSF, TGF- $\beta$, Stanniocalcin-1, and IGF-I), angiogenesis (VEGF, MCP-1, and IGF-I), local stem and progenitor cell growth and differentiation support (SCF, Angiopoietin-1, and SDF-1), antifibrosis (HGF and bFGF), and chemoattraction (CCL2, CCL4, and CXCL12) [7]. Additionally, beneficial effects of the use of MSC conditioned media (CM) have been reported; even $\mathrm{CM}$ has been shown to be therapeutically better than the cells themselves $[8,9]$.

However, although these properties are generally attributed to all MSCs derived from different tissues, evidence from different studies has suggested that MSCs from diverse sources are not identical and do not always achieve the same efficacy levels and desired outcomes. Likewise, diverse donor conditions can affect the MSC characteristics because the environment "niche" in which MSCs are immersed may be affected. In this review, we will describe some of the biological characteristics of MSCs that must be considered and the effects of the disease status of donors and recipients on these characteristics.

\section{Biological Characteristics}

2.1. Phenotypic Profile. Since Friedenstein and colleagues first isolated a colony-forming unit fibroblast (CFU-F) from bone marrow (BM) [10], bone marrow has been widely used as a source of MSCs for many investigations and clinical trials. In addition to bone marrow, MSCs have been isolated from different tissues such as adipose tissue [11], umbilical cord blood [12], dental pulp [13], synovial liquid and amniotic fluid $[14,15]$. All these tissues vary in their cellular components, signals, and factors secreted, resulting in different immediate microenvironment conditions, thus developing several physiological niches. Although isolated and long-term cultured MSCs of most tissues show similar immunophenotypic characteristics, some differences have been found among MSCs of different tissue origins according to data obtained by in vitro experiments. In 2006, the International Society of Cellular Therapy (ISCT) published the minimal criteria to define MSCs by nomenclature (Table 1) and by biological characteristics [10, 16-22] to allow studies from different groups to be compared and contrasted. These criteria include the following: (i) coexpression of markers such as CD73, CD90, and CD105 and a lack of expression of haematopoietic markers (CD45, CD34, and CD14) and human leucocyte antigen (HLA-DR), (ii) multipotent differentiation potential, and (iii) adherence to plastic. However, several researchers have noted that adipose-tissue-derived MSCs (AD-MSCs) express CD34 and CD54 in early passages [23] and have lower expression of CD106 and that umbilical cord bloodderived MSCs (UCB-MSCs) express CD90 and CD105 [24]. Other markers have been used in different studies, and other differences have emerged, such as VEGFR-2 (Flk-1) expression, which was significantly higher in periosteumderived cells compared to that in adipose tissue- and musclederived cells, or the rate of NGFR positivity, which was much higher in muscle-derived cells compared to that in other mesenchymal tissue-derived cells [25].

Although some immunophenotypic differences have been documented, many researchers consider the fact that these differences could be due to distinct extraction methods and different culture methodologies, resulting in variations of MSC surface markers. Thus, this review aimed to further investigate markers and characteristics that are more specific to select the better sources of MSCs for clinical applications.

Likewise, expanding the cells in vitro is necessary to obtain the desired numbers for therapeutic approaches. Changes in the proteomic phenotype of AD-MSCs have been observed during passages [26], although no proper approaches to examine the state of cells continuously during long-term in vitro culture have been established. Some researchers ascribe these variations to the adaptation of cells to the environment; thus, determining the biomolecular markers that are involved in these variations is essential for obtaining a better phenotypic characterisation of these cells and thus for achieving more effective cell therapy in the future.

2.2. MSC Proliferation. The proliferative activity of MSCs is another feature that may be affected by the different origins of MSCs. The rate and persistence of MSC proliferation appear to vary between source tissues. MSCs are considered adult stem cells, and, unlike embryonic stem cells (ESCs), these 
cells have a limited proliferative capacity. Physiological niches maintain adult stem cells in an undifferentiated state; however, when MSCs are cultured in vitro, they age, which affects their therapeutic properties, such as alterations in phenotype, differentiation potential, global gene expression patterns, miRNA profiles [27], and even chromosomal abnormalities [28], particularly after long-term culture or when cells of multiple doublings are used. Large numbers of MSCs are needed for therapeutic applications, and in vitro expansion is required to produce the desired MSC numbers. In vivo, MSCs represent $0.0001 \%$ of nucleated $\mathrm{BM}$ cells, and their number decreases with the age of the donor. The quantity of MSCs (CFU-Fs) among nucleated BM cells decreases with age from one MSC in $10^{4} \mathrm{BM}$ cells in newborns to one MSC in $10^{5}$ cells in teenagers and to one MSC in $10^{6}$ cells in older individuals [29]. Furthermore, MSCs from older human donors differ significantly from those from younger donors in morphology, replicative lifespan [30], doubling time, healing capacity [31], and differentiation potential. Sufficient evidence has indicated that MSCs from older donors have limited therapeutic efficacy, and some studies have suggested that the difference between preclinical and clinical findings is due to the donor age. Therefore, considering that several age-related diseases exist and that elderly patients are potential users of cell therapy, understanding the molecular and biological effects of ageing on MSCs is essential for developing safe and effective MSC-based autologous cell therapy. Meanwhile, the use of allogeneic MSCs may be a treatment option for these specific patients. As we comment below, MSCs elude allogeneic rejection, and their infusion is feasible and well tolerated, with no adverse effects $[32,33]$.

2.3. Differentiation Capacity. MSCs have the ability to differentiate in vitro into several mesenchymal lineages including adipose tissue, bone, cartilage, and muscle [15, 34, 35]. Furthermore, MSCs can differentiate into endothelial cells, neurons, and glial cells because MSCs express genes related to specific lineages rather than to those of the mesenchymal lineage [36]. Although multilineage differentiation is another minimal criterion advised by the ISCT and undoubtedly represents a fundamental property of MSCs, this ability depends primarily on the source tissue from which these cells are derived. As discussed by Sakaguchi et al. [25], who compared human MSCs isolated from bone marrow, synovium, periosteum, skeletal muscle, and adipose tissue and expanded them by similar processes, synovium-derived cells have the greatest ability for chondrogenesis; adipose- and synovium-derived cells have the greatest ability for adipogenesis; and bone marrow-, synovium-, and periosteum-derived cells have the greatest ability for osteogenesis. In another comparative analysis, UCB-MSCs showed no adipogenic differentiation capacity in contrast to BM- and AT-MSCs [37]. As discussed by Horwitz et al. [38], who used differentiated MSCs in a study to test the regeneration of damaged tissues, BM-MSCs can engraft after transplantation, differentiate to functional osteoblasts and contribute to the formation of new dense bone in children with osteogenesis imperfecta. Most likely, the microenvironment in which MSCs are transplanted directly influences their distinct differentiation pathways.
New insights into the biological characteristics of MSCs are needed to achieve future therapies.

2.4. Immunomodulatory Actions. Immunomodulatory properties of MSCs and their immunoprivileged condition make these cells good candidates for use in several clinical trials related to chronic, inflammatory, and autoimmune diseases. MSCs interact with cells of the innate or adaptive immune system (T cells, B cells, NK cells, monocyte-derived dendritic cells, and neutrophils) $[39,40]$. For a cell to be recognised by the immune system, the expression of major histocompatibility complex (MHC) and costimulatory molecules is necessary. MHC class I and class II human leukocyte antigens (HLAs) are master triggers of robust immunological rejection of grafts because they present antigens to cytolytic T lymphocytes (CTL) [41]. Human mesenchymal stem cells (hMSCs) are characterised by low expression of MHC class I HLAs but are constitutively negative for class II HLCs; these cells do not express costimulatory molecules such as B7-1, B7-2, CD80, CD86, CD40, and CD40L [42]. However, similar to the thymic epithelium, MSCs express the surface markers VCAM-1, ICAM-2, and LFA-3 [42, 43], which are crucial for $\mathrm{T}$ cell interactions. Although a $\mathrm{T}$ cell response should be expected, hMSCs are able to modulate the activation and proliferation of both $\mathrm{CD} 4+$ and CD8+ cells in vitro by arresting $\mathrm{T}$ cells in G0/G1 phase [44, 45]. Different studies have suggested that cell-cell interactions and certain soluble factors are the mechanisms used by MSCs to mediate the immune response. Factors such as IDO, TGF- $\beta 1$, IFN- $\gamma$, IL$1 \beta$, TNF $\alpha$, IL-6, IL-10, PGE-2, HGF, and HLA-G5 are secreted by MSCs or released after interactions with target cells. As we mentioned above, MSCs remain in a resting state, display antiapoptotic properties and maintain different cells such as haematopoietic stem cells (HSCs), thus contributing to tissue homeostasis. However, in an inflammatory environment such as that created by cytokines such as IFN- $\gamma$, TNF- $\alpha$, IL- $1 \alpha$, and IL-1 $\beta$, MSCs begin to exert their immunosuppressive effects and polarise, inhibiting the proliferation of effector cells and their production of cytokines. In this regard, IFN- $\gamma$ is postulated as a "licensing" agent for MSC antiproliferative action. MSCs may also acquire behaviour as antigen-presenting cells (APCs) under certain concentrations of IFN- $\gamma[46,47]$. However, no consensus regarding what concentration of IFN- $\gamma$ is more necessary for MSCs to show that inhibitory or APC functions exists. Likewise, TNF- $\alpha$ is another proinflammatory cytokine involved in the MSC immune response, and TNF- $\alpha$ enhances the effect of IFN- $\gamma$ [48]. IFN- $\gamma$, with or without the help of TNF- $\alpha$, stimulates the production of IDO by MSCs, inhibiting the proliferation of activated T or NK cells [49] and thus enhancing the homing potential and reparative properties of these cells; however, some potential risks are associated with the role of IFN- $\gamma$ [50].

Some authors have maintained that the immunomodulatory properties of MSCs are comparable [51,52], while others have argued that MSCs of different tissue origins or species cannot have equivalent immunomodulatory properties [53, 54]. For example, MSCs from perinatal sources (umbilical cord and amniotic membrane) show a higher immunomodulatory capacity, differential gene expression profiles, and 
paracrine factor secretion compared to BM-MSCs [55]. Interestingly, in 2012, Lee and colleagues found that HLA-G, a specific MHC-I antigen that is critical for maintaining the immune-tolerant state of pregnancy and that is a contributing factor to the induction of stronger immunosuppression [56], is strongly positive only in placenta-derived MSCs (PDMSCs) in contrast to BM-MSCs and AD-MSCs, suggesting that the immunophenotype of PD-MSCs may be superior to other MSCs in terms of their immunosuppressive function [57]. Nevertheless, in another related study, BM-MSCs were more immunomodulatory than PD-MSCs [58]. Melief et al. [59] concluded that the immunomodulatory capacities of BM-MSCs and AD-MSCs are similar but that differences in cytokine secretion cause $\mathrm{AD}-\mathrm{MSCs}$ to have more potent immunomodulatory effects than BM-MSCs.

A 2002 study showed that allogeneic MSCs prolonged skin graft survival in baboons [60]. Mouse MSCs have been used in related experiments; these cells use inducible nitric oxide synthase (iNOS) for immunosuppression instead of IDO. These findings indicate that MSCs differ between species [61]. Since then, several preclinical models have been used to analyse the biological effects of MSCs and their ability to modulate immune responses, considering that not all animal models mimic human diseases.

Once more, these differences could be due to isolation procedures, to culture methodology, or, more likely, to differences in the microenvironments where cells reside. These and other findings lead us to believe that determining whether these differences may be relevant for their clinical applications and whether MSCs of a particular tissue type are more appropriate for specific therapies or diseases is important.

\section{Preclinical Applications}

Preclinical models are essential for clinicians, researchers, and both national and international regulatory agencies to demonstrate the safety and efficacy of MSC-based therapies [62]. Because MSCs are able to exert immunomodulatory properties and to act on different immune cells both in vitro and in vivo as mentioned above, these cells have begun to be used against autoimmune diseases based on various autoimmune experimental models. Pioneer studies in experimental autoimmune encephalomyelitis (EAE), a model for multiple sclerosis, reported that MSCs derived from various tissue origins show efficacy against neurodegenerative disorders [63-68]. BM-MSC and UCB-MSC treatments have brought about improvements in clinical and laboratory parameters in systemic lupus erythematosus (SLE) [33, 69]. Furthermore, ameliorating effects have been observed in experimental mouse models of rheumatoid arthritis (RA) [70]. Diabetes is another autoimmune disorder in which MSCs have been employed [71-73]. Although promising results and progress have been observed in this field, the interspecies differences and contradictory experimental outcomes, as well as the inability to recreate the complete pathophysiology of some diseases, make it necessary to search for new animal models to yield comparable results.

\section{Autoimmune Diseases}

MSCs are being used to facilitate the engraftment of transplanted HSCs and to treat graft-versus-host disease (GVHD) after allogeneic haematopoietic stem cell transplantation (HSCT) based on their immunomodulatory properties and their ability to provide appropriate conditions; however, preclinical and clinical experiments with MSCs do not always show similar results for the prevention and treatment of GVHD. In a study using a mouse model of GVHD [74], MSCs suppressed alloantigen-induced $\mathrm{T}$ cell proliferation in vitro in a dose-dependent manner but yielded no clinical benefit regarding the incidence or severity of GVHD. Instead, when UCB-MSCs were administered in weekly doses in a xenogeneic model of GVHD, a marked decrease in human $\mathrm{T}$ cell proliferation was observed, and none of the mice developed GVHD. No therapeutic effect was obtained when UCB-MSCs were administered at the onset of GVHD [75]. In the same line of research, serial infusions of mouse AD-MSCs could efficiently control the lethal GVHD that occurred in recipients transplanted with haploidentical haematopoietic grafts [76]. Mixed results have also been achieved in human patients. One study found that the cotransplantation of culture-expanded MSCs and HSCs from HLA-identical sibling donors after myeloablative therapy accelerated haematopoietic engraftment [77]; however, a significant reduction of GVHD symptoms was not shown, although the incidence or severity of GVHD did not increase. Koç et al. [78] reported a positive impact of MSCs on haematopoiesis; rapid haematopoietic recovery was observed in a clinical study with breast cancer patients who received autologous HSCT together with autologous MSCs. Therapeutic effects have also been reported at the onset of GVHD, such as the case of a 9-year-old boy with severe treatment-resistant GVHD after allogeneic HSCT for acute lymphocytic leukaemia who received haploidentical MSCs derived from his mother. He showed improvement after 2 MSC administrations [79]. Similar results have been obtained in steroid-refractory GVHD pilot studies with BMMSCs and AD-MSCs [80, 81]. Several infusions appear to be required to maintain the level of active immunomodulation by MSCs. Similarly, the expression of proinflammatory cytokines such as IFN- $\gamma$ in the environment at the time of MSC administration is required by these cells to exert their immunosuppressive effect because a lack of MSC "licensing" can result in the absence of the desired therapeutic effect.

While evidence that MSCs are effective in combination or after HSCT in specific haematological and nonhaematological diseases has been shown, adverse reactions and risk factors intrinsic to this practice have been reported. In a pilot study, HLA-identical sibling-matched HSCs were transplanted with or without MSCs in haematological malignancy patients. Although MSCs were well tolerated and this treatment effectively prevented GVHD, six patients $(60 \%)$ in the MSC group and three (20\%) in the non-MSC group had 3year disease-free survival rates of 30 and $66.7 \%$, respectively [82]. The relapse rate in the experimental group was higher than that in the control group, suggesting that MSCs may impair the therapeutic graft-versus-leukaemia (GVL) effect. 
In vitro and in vivo studies regarding the relationship between the immunosuppressive properties of MSCs and the stimulation of cancer growth have been performed. Mouse MSCs from $\mathrm{BM}$, spleen, and thymus that were injected together with a genetically modified tumour cell vaccine could equally prevent the onset of an antitumour memory immune response, thus leading to tumour growth in normally resistant mice [83]. In another in vivo experiment with a murine melanoma tumour model, the authors observed that the subcutaneous injection of B16 melanoma cells led to tumour growth in allogeneic recipients only when MSCs were coinjected [84]. The functions of MSCs can be influenced by the existing microenvironment, making them acquire supportive properties towards cancer cells and decrease immune reactions [85]. Therefore, potential risks, related to the growth support and enhancement of undetected or "resident" cancer, do exist, and the administration of MSCs in these patients must be thoroughly evaluated.

\section{Do MSCs Carry out the Patient's Disease?}

One of the strategies to obtain MSCs for therapeutic purposes is an autologous approach. These cells are collected from patients by more or less invasive methods, isolated, seeded in culture under good manufacturing practice (GMP) quality standards, and reinjected into the patient. Nevertheless, when the repair mechanisms of the body are insufficient or ineffective, this treatment results in a homeostatic imbalance in the organism, producing degradation and disease and compromising the pool of endogenous cells, thus resulting in low efficacy. Some diseases provoke changes in the bone marrow microenvironment, which is one of the primary sources of MSCs, thus producing changes in the endogenous pool of MSCs and altering their biological features [86]. MSCs from patients with acute myeloid leukaemia showed abnormal biological properties, including morphological heterogeneity, limited proliferation capacity, and impaired differentiation and haematopoiesis support ability [87]. MSCs derived from patients with multiple myeloma showed impaired immuneinhibitory effects on $\mathrm{T}$ cells, decreasing their osteogenic potential [88]. Poor proliferation, differentiation potentials, and cytokine release defects were found in BM-MSCs derived from patients with aplastic anaemia, another haematopoietic disorder $[89,90]$.

Although the mechanisms remain unknown, MSCs appear to be involved in autoimmune pathologies. For instance, MSCs derived from patients with autoimmune diseases display the following altered functions. (i) MSCs from rheumatoid arthritis (RA) patients have an impaired ability to support haematopoiesis [91] and lower proliferative and clonogenic potentials [92]. (ii) MSCs from immune thrombocytopenic purpura (ITP) patients have a reduced proliferative capacity and a lower inhibitory effect on $\mathrm{T}$ cell proliferation compared with MSCs from healthy donors [93]. (iii) MSCs from systemic lupus erythematosus (SLE) patients display deficient growth, abnormal morphology, and upregulated telomerase activity $[94,95]$. (iv) MSCs from systemic sclerosis (SSc) patients display early senescence
[96]. In metabolic diseases such as diabetes, alterations in autologous MSCs have also been documented. A study using MSCs from type 2 diabetic mice showed that the number of these cells was diminished and that their proliferation and survival abilities were impaired in vitro. Moreover, diabetic MSC engraftment produced limited improvement in the diabetic subjects and could not produce the same therapeutic outcomes as in their nondiabetic counterparts in vivo [97]. Advanced glycation end products (AGEs) accumulate in the tissues of aged people, and these products are involved in diabetes and in musculoskeletal diseases. In 2005, Kume et al. [98] investigated the effect of AGEs on MSCs and showed that AGEs inhibited MSC proliferation, induced MSC apoptosis, and interfered with MSC differentiation into adipose tissue, cartilage, and bone. Another study examined type 2 diabetesderived AD-MSCs and found that these cells had functional impairments in their multilineage potential and proliferative capacity because of prolonged exposure to high glucose concentrations [99]. We demonstrated that diabetic-derived $\mathrm{AD}$-MSCs have an altered phenotype related to plasminogen activator inhibitor-1 (PAI-1) expression levels and display reduced fibrinolytic activity [100]. In this respect, our preliminary results and others suggest that the immunogenicity of MSCs could have related effects on the coagulation system $[101,102]$. Thus, MSC-based therapy could lead to thrombotic events in particular recipients.

Although the possibility of healing with our own cells is extremely attractive, little is known regarding the influence of different disease states and concomitant medications on MSCs [103, 104]. Thus, although the use of autologous MSCs for cell therapy is widespread, their use in humans must be handled with extreme caution. Researching and analysing both the risks and benefits of this therapy in individual patients and for each disease state are necessary.

\section{Safety and Efficacy in Clinical Trials}

Several clinical trials are in progress to ensure the safety and efficacy of MSCs used as medicaments. For cell-based products, we must consider that cells are living products and that their interactions with body fluids remain unclear $[100,102,105]$.

Phase I clinical trials are the first step in the investigation of a new drug and include pharmacokinetic and pharmacodynamic studies in which the patient's safety plays an essential role in the development of medicaments. The primary goal of phase II clinical trials is to provide preliminary information regarding the drug efficacy and safety supplement data obtained in phase I trials. Usually, safety evaluations are based on possible complications derived from the procedure in a time-dependent manner after the administration of the cells. Efficacy parameters focus on the improvement of clinical effects at a given time. MSC-based cell therapy is a relatively new therapeutic option for certain diseases, and data regarding the long-term monitoring of patients remain lacking. Nevertheless, the administration of MSCs is considered a feasible and safe procedure with no adverse events reported. However, the risks associated with stem cell 


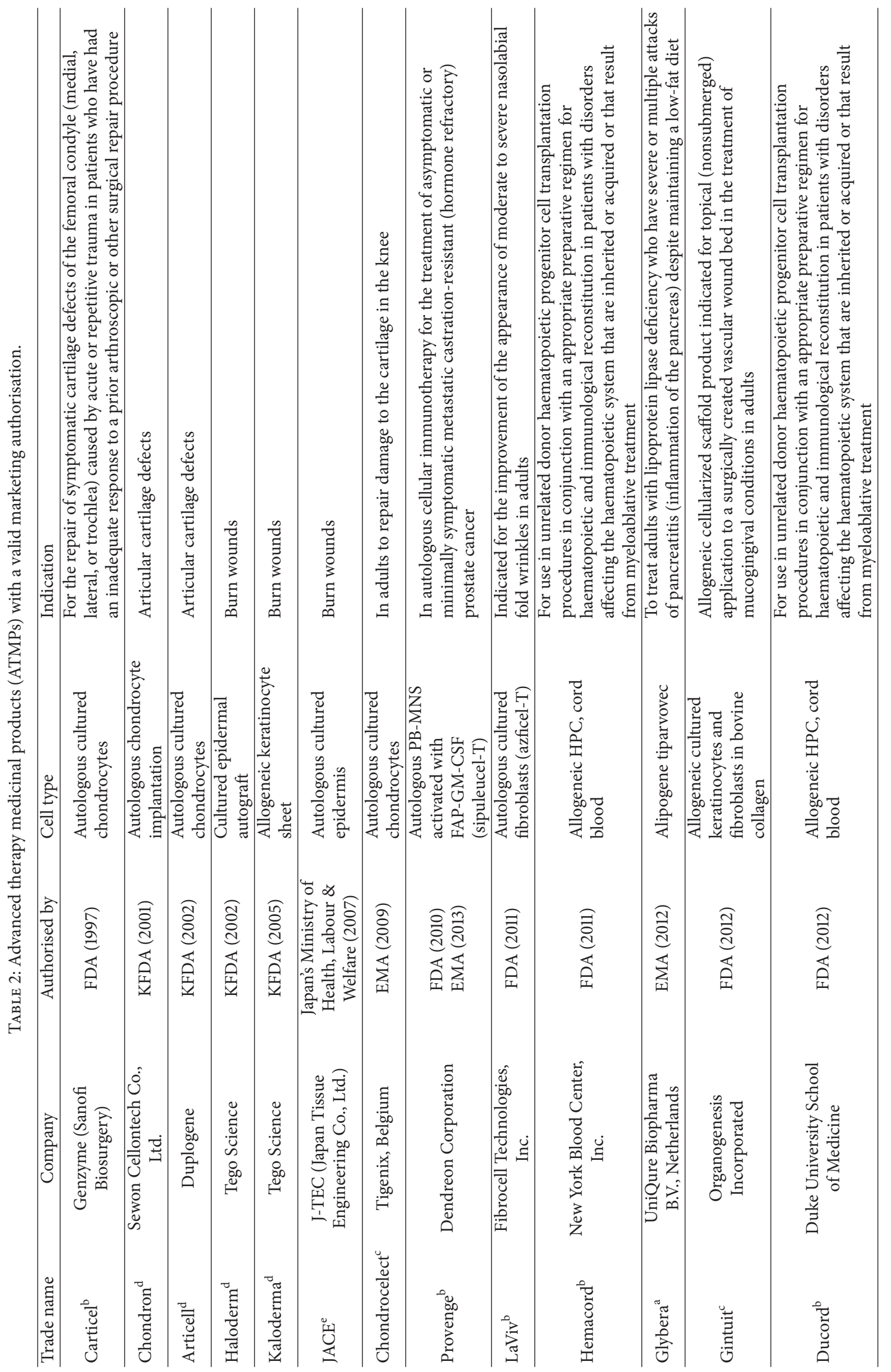




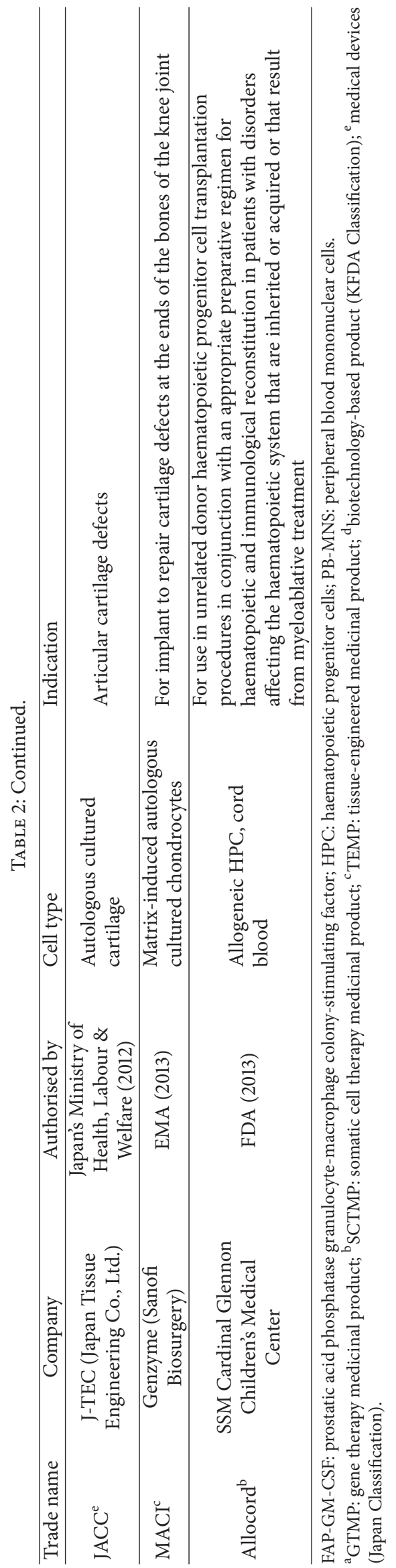


TABLE 3: MSC cell-based therapies with a valid marketing authorisation.

\begin{tabular}{|c|c|c|c|c|}
\hline Trade name & Company & Authorised by & Cell type & Indication \\
\hline Hearticellgram* & FCB PharmiCell & KFDA (2011) & Autologous BM-derived MSCs & $\begin{array}{l}\text { Treatment for postacute } \\
\text { myocardial infarction }\end{array}$ \\
\hline Cartistem $^{*}$ & Medipost & KFDA (2012) & Allogeneic hUCB-MSCs & $\begin{array}{l}\text { Treatment of traumatic and } \\
\text { degenerative osteoarthritis }\end{array}$ \\
\hline Prochymal $^{*}$ & Osiris Therapeutics Inc. & $\begin{array}{c}\text { Health Canada (2012) } \\
\text { New Zealand (2012) }\end{array}$ & Allogeneic BM-MSCs & $\begin{array}{l}\text { Treatment of acute GvHD } \\
\text { children who are unresponsive to } \\
\text { steroids }\end{array}$ \\
\hline
\end{tabular}

MSCs: mesenchymal stem cells; BM: bone marrow; hUCB: human umbilical cord blood; GvHD: graft-versus-host disease; KFDA: Korean Food and Drug Administration.

*SCTMP: somatic cell therapy medicinal product.

therapy [106] must be considered because these risks increase the probability of the occurrence of an adverse event. The cell source, donor origin, product manufacturing, and recipient disease status are important factors related to the safety and efficacy of the use of MSCs. In this regard, the use of bovine proteins in the medium used to culture these cells [38] and the observed formation of ectopic tissue in animal models $[107,108]$, as well as malignant transformation $[109,110]$ and immune responses, must be evaluated before wider clinical applications and registration are accepted.

\section{Clinical Manufacturing of MSC-Based Medicines}

With the exception of haematopoietic stem cell transplants, stem cell therapies used for the treatment of any disease are considered drugs; therefore, their development, approval, and use must be in accordance with specific standards established for such medicines nationally and internationally. MSCs are called advanced therapy medicinal products (ATMPs) and are under regulation number 1394/2007. Relating production processes and development staff, clinicians, and researchers is obligatory to achieve GMP procedures under European regulations [111,112]. Currently, no standardised manufacturing platform exists, although most facilities employ standard release criteria to measure sterility, viability, and chromosomal stability to meet European or FDA regulations [113]. Although regulation establishes common parameters to follow, different protocols are used to isolate these cells, and the processes, plating densities, and reagents used cause the results to differ from each other. Donor selection in terms of age and disease status is another variable to consider due to known MSC donor-to-donor heterogeneity [114]. The cell source is another important factor related to the efficacy of the product. As reported previously, MSCs derived from different tissues do not always achieve the same level of efficacy. Additionally, culture media used for the production of MSCs could affect the basic characteristics of cells; thus, designing a fully defined medium free of animal and human origins is crucial.

Thus far, no MSC-based medicine product has marketing authorisation in the European Union, although four gene and cell-based products have a valid marketing authorisation awarded by the European Medicines Agency. However, since
2011, three MSC products have received marketing approval in other regions [115] (Tables 2 and 3).

The MSC field continues its upward progression, with a growing number of established companies and ongoing clinical trials, but remaining challenges must be overcome. Bottlenecks exist regarding donor selection, cells sources, isolation protocols, culture media used, open-culture systems, bioreactors, and recipient disease status. Establishing a standardised and comparable process is also crucial to ensure biological and functional equivalence between product lots.

\section{Concluding Remarks and Future Perspectives}

Treatments based on the use of human stem cells are novel and promising therapeutic alternatives for some diseases. Spain is at the forefront of research using such treatments, and these treatments are developed and evaluated with great scientific rigor. Currently, the use of living cells as a medicinal product is becoming realistic. Cell therapy should be safe, pure, stable and efficient. Cell-based products are more complex and depend on the physiological and genetic heterogeneity of the patient. Obtaining as much information as possible with the tools we have at our disposal is essential for ensuring the safety, reliability, quality, and effectiveness of the manufactured product. MSCs are leading the way into a new era of regenerative medicine, and their multifaceted features make them powerful candidates to become tools to treat several diseases. However, their indiscriminate use has resulted in mixed outcomes in preclinical and clinical studies. While MSCs derived from diverse tissues share some common properties, they markedly differ in terms of their differentiation abilities, growth rates, healing capacity, and gene expression profile. Similarly, the disease statuses of donors and recipients are important factors to consider when using MSCs as therapeutic agents because factors such as the MSC behaviour with body fluids and specific disease environments remain unclear. Available data suggest that some tissue-specific MSCs are more appropriate than others according to particular pathologies. Equally, some evidence has indicated that certain patient profiles are not suitable to be treated with these therapies. Thus, multiple bottlenecks for the standardisation of therapeutic protocols exist. Future well-designed clinical trials and long-term monitoring of 
patients are crucial for obtaining additional information regarding the therapeutic use of MSCs.

\section{Conflict of Interests}

The authors declare that no conflict of interests regarding the publication of this paper exists.

\section{Acknowledgments}

The authors are supported by the Fundación Progreso y Salud, Consejería de Salud, Junta de Andalucía; FEDER cofunded grants from Consejería de Innovación Ciencia y Empresa, Junta de Andalucía (Grants CTS-6505; INP-20111615-900000); FEDER cofunded grants from Instituto de Salud Carlos III (Red TerCel-Grant RD12/0019/0028; PI10/ 00964 and PI14/01015) and the Ministry of Health and Consumer Affairs (Advanced Therapies Program Grant TRA120); SUDOE Program-BIOREG (Regenerative Medicine Network-SOE3/P1/E750) and ACTION Cost (European Cooperation in Science and Technology-BM1305). Support from FSED and FAID allowed access to databanks. CIBERDEM is an initiative of the Instituto de Salud Carlos III.

\section{References}

[1] Y. Jiang, B. N. Jahagirdar, R. L. Reinhardt et al., "Pluripotency of mesenchymal stem cells derived from adult marrow," Nature, vol. 418, no. 6893, pp. 41-49, 2002.

[2] L. da Silva Meirelles, P. C. Chagastelles, and N. B. Nardi, "Mesenchymal stem cells reside in virtually all post-natal organs and tissues," Journal of Cell Science, vol. 119, no. 11, pp. 22042213, 2006.

[3] A. I. Caplan, "All MSCs are pericytes?" Cell Stem Cell, vol. 3, no. 3, pp. 229-230, 2008.

[4] D. J. Prockop and J. Y. Oh, "Mesenchymal stem/stromal cells (MSCs): role as guardians of inflammation," Molecular Therapy, vol. 20, no. 1, pp. 14-20, 2012.

[5] R. J. Petrie, A. D. Doyle, and K. M. Yamada, "Random versus directionally persistent cell migration," Nature Reviews Molecular Cell Biology, vol. 10, no. 8, pp. 538-549, 2009.

[6] M. J. Hoogduijn, F. Popp, R. Verbeek et al., "The immunomodulatory properties of mesenchymal stem cells and their use for immunotherapy," International Immunopharmacology, vol. 10, no. 12, pp. 1496-1500, 2010.

[7] L. da Silva Meirelles, A. M. Fontes, D. T. Covas, and A. I. Caplan, "Mechanisms involved in the therapeutic properties of mesenchymal stem cells," Cytokine and Growth Factor Reviews, vol. 20, no. 5-6, pp. 419-427, 2009.

[8] C. Shrestha, L. Zhao, K. Chen, H. He, and Z. Mo, "Enhanced healing of diabetic wounds by subcutaneous administration of human umbilical cord derived stem cells and their conditioned media," International Journal of Endocrinology, vol. 2013, Article ID 592454, 10 pages, 2013.

[9] A. Burlacu, G. Grigorescu, A.-M. Rosca, M. B. Preda, and M. Simionescu, "Factors secreted by mesenchymal stem cells and endothelial progenitor cells have complementary effects on angiogenesis in vitro," Stem Cells and Development, vol. 22, no. 4, pp. 643-653, 2013.
[10] A. J. Friedenstein, U. F. Deriglasova, N. N. Kulagina et al., "Precursors for fibroblasts in different populations of hematopoietic cells as detected by the in vitro colony assay method," Experimental Hematology, vol. 2, no. 2, pp. 83-92, 1974.

[11] P. A. Zuk, M. Zhu, H. Mizuno et al., "Multilineage cells from human adipose tissue: implications for cell-based therapies," Tissue Engineering, vol. 7, no. 2, pp. 211-228, 2001.

[12] A. Erices, P. Conget, and J. J. Minguell, "Mesenchymal progenitor cells in human umbilical cord blood," British Journal of Haematology, vol. 109, no. 1, pp. 235-242, 2000.

[13] S. Gronthos, M. Mankani, J. Brahim, P. G. Robey, and S. Shi, "Postnatal human dental pulp stem cells (DPSCs) in vitro and in vivo," Proceedings of the National Academy of Sciences of the United States of America, vol. 97, no. 25, pp. 13625-13630, 2000.

[14] S. Díaz-Prado, E. Muiños-López, T. Hermida-Gómez et al., "Multilineage differentiation potential of cells isolated from the human amniotic membrane," Journal of Cellular Biochemistry, vol. 111, no. 4, pp. 846-857, 2010.

[15] M. F. Pittenger, "Multilineage potential of adult human mesenchymal stem cells," Science, vol. 284, no. 5411, pp. 143-147, 1999.

[16] M. Owen and A. J. Friedenstein, "Stromal stem cells: marrowderived osteogenic precursors," Ciba Foundation Symposium, vol. 136, pp. 42-60, 1988.

[17] A. I. Caplan, "Mesenchymal stem cells," Journal of Orthopaedic Research, vol. 9, no. 5, pp. 641-650, 1991.

[18] J. E. Dennis, A. Merriam, A. Awadallah, J. U. Yoo, B. Johnstone, and A. I. Caplan, "A quadripotential mesenchymal progenitor cell isolated from the marrow of an adult mouse," Journal of Bone and Mineral Research, vol. 14, no. 5, pp. 700-709, 1999.

[19] P. Bianco and P. G. Robey, "Marrow stromal stem cells," Journal of Clinical Investigation, vol. 105, no. 12, pp. 1663-1668, 2000.

[20] Y. Jiang, B. Vaessen, T. Lenvik, M. Blackstad, M. Reyes, and C. M. Verfaillie, "Multipotent progenitor cells can be isolated from postnatal murine bone marrow, muscle, and brain," Experimental Hematology, vol. 30, no. 8, pp. 896-904, 2002.

[21] M. Dominici, K. Le Blanc, I. Mueller et al., "Minimal criteria for defining multipotent mesenchymal stromal cells. The International Society for Cellular Therapy position statement," Cytotherapy, vol. 8, no. 4, pp. 315-317, 2006.

[22] A. I. Caplan, "What's in a name?" Tissue Engineering, Part A, vol. 16, no. 8, pp. 2415-2417, 2010.

[23] D. A. de Ugarte, Z. Alfonso, P. A. Zuk et al., "Differential expression of stem cell mobilization-associated molecules on multi-lineage cells from adipose tissue and bone marrow," Immunology Letters, vol. 89, no. 2-3, pp. 267-270, 2003.

[24] M. Krampera, M. Franchini, G. Pizzolo, and G. Aprili, "Mesenchymal stem cells: from biology to clinical use," Blood Transfusion, vol. 5, no. 3, pp. 120-129, 2007.

[25] Y. Sakaguchi, I. Sekiya, K. Yagishita, and T. Muneta, "Comparison of human stem cells derived from various mesenchymal tissues: superiority of synovium as a cell source," Arthritis \& Rheumatism, vol. 52, no. 8, pp. 2521-2529, 2005.

[26] E. Capra, R. Beretta, V. Parazzi et al., "Changes in the proteomic profile of adipose tissue-derived mesenchymal stem cells during passages," Proteome Science, vol. 10, no. 1, article 46, 2012.

[27] W. Wagner, S. Bork, G. Lepperdinger et al., "How to track cellular aging of mesenchymal stromal cells?” Aging, vol. 2, no. 4, pp. 224-230, 2010.

[28] K. Tarte, J. Gaillard, J.-J. Lataillade et al., "Clinical-grade production of human mesenchymal stromal cells: occurrence 
of aneuploidy without transformation," Blood, vol. 115, no. 8, pp. 1549-1553, 2010.

[29] A. I. Caplan, "Why are MSCs therapeutic? New data: new insight," The Journal of Pathology, vol. 217, no. 2, pp. 318-324, 2009.

[30] K. Stenderup, J. Justesen, C. Clausen, and M. Kassem, "Aging is associated with decreased maximal life span and accelerated senescence of bone marrow stromal cells," Bone, vol. 33, no. 6, pp. 919-926, 2003.

[31] R. Schäfer, U. Knauf, M. Zweyer et al., "Age dependence of the human skeletal muscle stem cell in forming muscle tissue," Artificial Organs, vol. 30, no. 3, pp. 130-140, 2006.

[32] D. F. McAuley, G. F. Curley, U. I. Hamid et al., "Clinical grade allogeneic human mesenchymal stem cells restore alveolar fluid clearance in human lungs rejected for transplantation," The American Journal of Physiology-Lung Cellular and Molecular Physiology, vol. 306, no. 9, pp. L809-L815, 2014.

[33] J. Liang, H. Zhang, B. Hua et al., "Allogenic mesenchymal stem cells transplantation in refractory systemic lupus erythematosus: a pilot clinical study," Annals of the Rheumatic Diseases, vol. 69, no. 8, pp. 1423-1429, 2010.

[34] D. J. Prockop, "Marrow stromal cells as stem cells for nonhematopoietic tissues," Science, vol. 276, no. 5309, pp. 71-74, 1997.

[35] S. P. Bruder, N. Jaiswal, and S. E. Haynesworth, "Growth kinetics, self-renewal, and the osteogenic potential of purified human mesenchymal stem cells during extensive subcultivation and following cryopreservation," Journal of Cellular Biochemistry, vol. 64, no. 2, pp. 278-294, 1997.

[36] D. Woodbury, K. Reynolds, and I. B. Black, "Adult bone marrow stromal stem cells express germline, ectodermal, endodermal, and mesodermal genes prior to neurogenesis," Journal of Neuroscience Research, vol. 69, no. 6, pp. 908-917, 2002.

[37] S. Kern, H. Eichler, J. Stoeve, H. Klüter, and K. Bieback, "Comparative analysis of mesenchymal stem cells from bone marrow, umbilical cord blood, or adipose tissue," Stem Cells, vol. 24, no. 5, pp. 1294-1301, 2006.

[38] E. M. Horwitz, P. L. Gordon, W. K. K. Koo et al., "Isolated allogeneic bone marrow-derived mesenchymal cells engraft and stimulate growth in children with osteogenesis imperfecta: implications for cell therapy of bone," Proceedings of the National Academy of Sciences of the United States of America, vol. 99, no. 13, pp. 8932-8937, 2002.

[39] M. D. Nicola, C. Carlo-Stella, M. Magni et al., "Human bone marrow stromal cells suppress T-lymphocyte proliferation induced by cellular or nonspecific mitogenic stimuli," Blood, vol. 99, no. 10, pp. 3838-3843, 2002.

[40] L. Raffaghello, G. Bianchi, M. Bertolotto et al., "Human mesenchymal stem cells inhibit neutrophil apoptosis: a model for neutrophil preservation in the bone marrow niche," Stem Cells, vol. 26, no. 1, pp. 151-162, 2008.

[41] A. Hmadcha, J. Domínguez-Bendala, J. Wakeman, M. Arredouani, and B. Soria, "The immune boundaries for stem cell based therapies: problems and prospective solutions," Journal of Cellular and Molecular Medicine, vol. 13, no. 8, pp. 1464-1475, 2009.

[42] K. Le Blanc, "Immunomodulatory effects of fetal and adult mesenchymal stem cells," Cytotherapy, vol. 5, no. 6, pp. 485-489, 2003.

[43] P. A. Conget and J. J. Minguell, "Phenotypical and functional properties of human bone marrow mesenchymal progenitor cells," Journal of Cellular Physiology, vol. 181, no. 1, pp. 67-73, 1999.

[44] S. Glennie, I. Soeiro, P. J. Dyson, E. W.-F. Lam, and F. Dazzi, "Bone marrow mesenchymal stem cells induce division arrest anergy of activated T cells," Blood, vol. 105, no. 7, pp. 2821-2827, 2005.

[45] F. Benvenuto, S. Ferrari, E. Gerdoni et al., "Human mesenchymal stem cells promote survival of T cells in a quiescent state," Stem Cells, vol. 25, no. 7, pp. 1753-1760, 2007.

[46] J. Stagg, S. Pommey, N. Eliopoulos, and J. Galipeau, "Interferon$\gamma$-stimulated marrow stromal cells: a new type of nonhematopoietic antigen-presenting cell," Blood, vol. 107, no. 6, pp. 2570-2577, 2006.

[47] A. Uccelli, L. Moretta, and V. Pistoia, "Mesenchymal stem cells in health and disease," Nature Reviews Immunology, vol. 8, no. 9, pp. 726-736, 2008.

[48] C. Menard, L. Pacelli, G. Bassi et al., "Clinical-grade mesenchymal stromal cells produced under various good manufacturing practice processes differ in their immunomodulatory properties: standardization of immune quality controls," Stem Cells and Development, vol. 22, no. 12, pp. 1789-1801, 2013.

[49] M. Krampera, L. Cosmi, R. Angeli et al., "Role for interferon$\gamma$ in the immunomodulatory activity of human bone marrow mesenchymal stem cells," Stem Cells, vol. 24, no. 2, pp. 386-398, 2006.

[50] K. N. Sivanathan, S. Gronthos, D. Rojas-Canales, B. Thierry, and P. T. Coates, "Interferon-gamma modification of mesenchymal stem cells: implications of autologous and allogeneic mesenchymal stem cell therapy in allotransplantation," Stem Cell Reviews and Reports, vol. 10, no. 3, pp. 351-375, 2014.

[51] M. Najar, G. Raicevic, H. I. Boufker et al., "Mesenchymal stromal cells use PGE2 to modulate activation and proliferation of lymphocyte subsets: combined comparison of adipose tissue, Wharton's Jelly and bone marrow sources," Cellular Immunology, vol. 264, no. 2, pp. 171-179, 2010.

[52] K. H. Yoo, I. K. Jang, M. W. Lee et al., "Comparison of immunomodulatory properties of mesenchymal stem cells derived from adult human tissues," Cellular Immunology, vol. 259, no. 2, pp. 150-156, 2009.

[53] M. Ricciardi, G. Malpeli, F. Bifari et al., "Comparison of epithelial differentiation and immune regulatory properties of mesenchymal stromal cells derived from human lung and bone marrow," PLoS ONE, vol. 7, no. 5, Article ID e35639, 2012.

[54] M. Krampera, "Mesenchymal stromal cell licensing: a multistep process," Leukemia, vol. 25, no. 9, pp. 1408-1414, 2011.

[55] H. Wegmeyer, A.-M. Bröske, M. Leddin et al., "Mesenchymal stromal cell characteristics vary depending on their origin," Stem Cells and Development, vol. 22, no. 19, pp. 2606-2618, 2013.

[56] J. S. Hunt, M. G. Petroff, R. H. McIntire, and C. Ober, "HLA-G and immune tolerance in pregnancy," The FASEB Journal, vol. 19, no. 7, pp. 681-693, 2005.

[57] J. M. Lee, J. Jung, H.-J. Lee et al., “Comparison of immunomodulatory effects of placenta mesenchymal stem cells with bone marrow and adipose mesenchymal stem cells," International Immunopharmacology, vol. 13, no. 2, pp. 219-224, 2012.

[58] H. Fazekasova, R. Lechler, K. Langford, and G. Lombardi, "Placenta-derived MSCs are partially immunogenic and less immunomodulatory than bone marrow-derived MSCs," Journal of Tissue Engineering and Regenerative Medicine, vol. 5, no. 9, pp. 684-694, 2011. 
[59] S. M. Melief, J. J. Zwaginga, W. E. Fibbe, and H. Roelofs, "Adipose tissue-derived multipotent stromal cells have a higher immunomodulatory capacity than their bone marrow-derived counterparts," Stem Cells Translational Medicine, vol. 2, no. 6, pp. 455-463, 2013.

[60] A. Bartholomew, C. Sturgeon, M. Siatskas et al., "Mesenchymal stem cells suppress lymphocyte proliferation in vitro and prolong skin graft survival in vivo," Experimental Hematology, vol. 30, no. 1, pp. 42-48, 2002.

[61] G. Ren, J. Su, L. Zhang et al., "Species variation in the mechanisms of mesenchymal stem cell-mediated immunosuppression," Stem Cells, vol. 27, no. 8, pp. 1954-1962, 2009.

[62] M. Krampera, J. Galipeau, Y. Shi, K. Tarte, and L. Sensebe, "Immunological characterization of multipotent mesenchymal stromal cells-the international society for cellular therapy (ISCT) working proposal," Cytotherapy, vol. 15, no. 9, pp. 10541061, 2013.

[63] E. Zappia, S. Casazza, E. Pedemonte et al., "Mesenchymal stem cells ameliorate experimental autoimmune encephalomyelitis inducing T-cell anergy," Blood, vol. 106, no. 5, pp. 1755-1761, 2005.

[64] D. Gordon, G. Pavlovska, C. P. Glover, J. B. Uney, D. Wraith, and N. J. Scolding, "Human mesenchymal stem cells abrogate experimental allergic encephalomyelitis after intraperitoneal injection, and with sparse CNS infiltration," Neuroscience Letters, vol. 448, no. 1, pp. 71-73, 2008.

[65] M. Rafei, E. Birman, K. Forner, and J. Galipeau, "Allogeneic mesenchymal stem cells for treatment of experimental autoimmune encephalomyelitis," Molecular Therapy, vol. 17, no. 10, pp. 1799-1803, 2009.

[66] G. Constantin, S. Marconi, B. Rossi et al., "Adipose-derived mesenchymal stem cells ameliorate chronic experimental autoimmune encephalomyelitis," Stem Cells, vol. 27, no. 10, pp. 26242635, 2009.

[67] L. Bai, D. P. Lennon, V. Eaton et al., "Human bone marrowderived mesenchymal stem cells induce Th2-polarized immune response and promote endogenous repair in animal models of multiple sclerosis," Glia, vol. 57, no. 11, pp. 1192-1203, 2009.

[68] J. Zhang, Y. Li, J. Chen et al., "Human bone marrow stromal cell treatment improves neurological functional recovery in EAE mice," Experimental Neurology, vol. 195, no. 1, pp. 16-26, 2005.

[69] L. Sun, D. Wang, J. Liang et al., "Umbilical cord mesenchymal stem cell transplantation in severe and refractory systemic lupus erythematosus," Arthritis and Rheumatism, vol. 62, no. 8, pp. 2467-2475, 2010

[70] M. A. González, E. González-Rey, L. Rico, D. Büscher, and M. Delgado, "Treatment of experimental arthritis by inducing immune tolerance with human adipose-derived mesenchymal stem cells," Arthritis and Rheumatism, vol. 60, no. 4, pp. 10061019, 2009.

[71] M. Jurewicz, S. Yang, A. Augello et al., "Congenic mesenchymal stem cell therapy reverses hyperglycemia in experimental type 1 diabetes," Diabetes, vol. 59, no. 12, pp. 3139-3147, 2010.

[72] P. Fiorina, M. Jurewicz, A. Augello et al., "Immunomodulatory function of bone marrow-derived mesenchymal stem cells in experimental autoimmune type 1 diabetes," The Journal of Immunology, vol. 183, no. 2, pp. 993-1004, 2009.

[73] R. H. Lee, M. J. Seo, R. L. Reger et al., "Multipotent stromal cells from human marrow home to and promote repair of pancreatic islets and renal glomeruli in diabetic NOD/scid mice," Proceedings of the National Academy of Sciences of the United States of America, vol. 103, no. 46, pp. 17438-17443, 2006.
[74] M. Sudres, F. Norol, A. Trenado et al., "Bone marrow mesenchymal stem cells suppress lymphocyte proliferation in vitro but fail to prevent graft-versus-host disease in mice," The Journal of Immunology, vol. 176, no. 12, pp. 7761-7767, 2006.

[75] V. Tisato, K. Naresh, J. Girdlestone, C. Navarrete, and F. Dazzi, "Mesenchymal stem cells of cord blood origin are effective at preventing but not treating graft-versus-host disease," Leukemia, vol. 21, no. 9, pp. 1992-1999, 2007.

[76] R. Yañez, M. L. Lamana, J. García-Castro, I. Colmenero, M. Ramírez, and J. A. Bueren, "Adipose tissue-derived mesenchymal stem cells have in vivo immunosuppressive properties applicable for the control of the graft-versus-host disease," Stem Cells, vol. 24, no. 11, pp. 2582-2591, 2006.

[77] H. M. Lazarus, O. N. Koc, S. M. Devine et al., "Cotransplantation of HLA-identical sibling culture-expanded mesenchymal stem cells and hematopoietic stem cells in hematologic malignancy patients," Biology of Blood and Marrow Transplantation, vol. 11, no. 5, pp. 389-398, 2005.

[78] O. N. Koç, S. L. Gerson, B. W. Cooper et al., "Rapid hematopoietic recovery after coinfusion of autologous-blood stem cells and culture-expanded marrow mesenchymal stem cells in advanced breast cancer patients receiving high-dose chemotherapy," Journal of Clinical Oncology, vol. 18, no. 2, pp. 307-316, 2000.

[79] K. Le Blanc, I. Rasmusson, B. Sundberg et al., "Treatment of severe acute graft-versus-host disease with third party haploidentical mesenchymal stem cells," The Lancet, vol. 363, no. 9419, pp. 1439-1441, 2004.

[80] O. Ringdén, M. Uzunel, I. Rasmusson et al., "Mesenchymal stem cells for treatment of therapy-resistant graft-versus-host disease," Transplantation, vol. 81, no. 10, pp. 1390-1397, 2006.

[81] B. Fang, Y. P. Song, L. M. Liao, Q. Han, and R. C. Zhao, "Treatment of severe therapy-resistant acute graft-versus-host disease with human adipose tissue-derived mesenchymal stem cells," Bone Marrow Transplantation, vol. 38, no. 5, pp. 389-390, 2006.

[82] H. Ning, F. Yang, M. Jiang et al., "The correlation between cotransplantation of mesenchymal stem cells and higher recurrence rate in hematologic malignancy patients: outcome of a pilot clinical study," Leukemia, vol. 22, no. 3, pp. 593-599, 2008.

[83] M. Krampera, S. Sartoris, F. Liotta et al., "Immune regulation by mesenchymal stem cells derived from adult spleen and thymus," Stem Cells and Development, vol. 16, no. 5, pp. 797-810, 2007.

[84] F. Djouad, P. Plence, C. Bony et al., "Immunosuppressive effect of mesenchymal stem cells favors tumor growth in allogeneic animals," Blood, vol. 102, no. 10, pp. 3837-3844, 2003.

[85] M. Galiè, G. Konstantinidou, D. Peroni et al., "Mesenchymal stem cells share molecular signature with mesenchymal tumor cells and favor early tumor growth in syngeneic mice," Oncogene, vol. 27, no. 18, pp. 2542-2551, 2008.

[86] B. Mazzanti, A. Aldinucci, T. Biagioli et al., "Differences in mesenchymal stem cell cytokine profiles between MS patients and healthy donors: implication for assessment of disease activity and treatment," Journal of Neuroimmunology, vol. 199, no. 1-2, pp. 142-150, 2008.

[87] Z. G. Zhao, Y. Liang, K. Li et al., "Phenotypic and functional comparison of mesenchymal stem cells derived from the bone marrow of normal adults and patients with hematologic malignant diseases," Stem Cells and Development, vol. 16, no. 4, pp. 637-648, 2007.

[88] B. Li, J. Fu, P. Chen, and W. Zhuang, "Impairment in immunomodulatory function of mesenchymal stem cells from multiple 
myeloma patients," Archives of Medical Research, vol. 41, no. 8, pp. 623-633, 2010.

[89] Y.-H. Chao, C.-T. Peng, H.-J. Harn, C.-K. Chan, and K.-H. $\mathrm{Wu}$, "Poor potential of proliferation and differentiation in bone marrow mesenchymal stem cells derived from children with severe aplastic anemia," Annals of Hematology, vol. 89, no. 7, pp. 715-723, 2010.

[90] A. Bacigalupo, M. Valle, M. Podestà et al., “T-cell suppression mediated by mesenchymal stem cells is deficient in patients with severe aplastic anemia," Experimental Hematology, vol. 33, no. 7, pp. 819-827, 2005.

[91] H. A. Papadaki, H. D. Kritikos, C. Gemetzi et al., "Bone marrow progenitor cell reserve and function and stromal cell function are defective in rheumatoid arthritis: evidence for a tumor necrosis factor alpha-mediated effect," Blood, vol. 99, no. 5, pp. 1610-1619, 2002.

[92] M.-C. Kastrinaki, P. Sidiropoulos, S. Roche et al., "Functional, molecular and proteomic characterisation of bone marrow mesenchymal stem cells in rheumatoid arthritis," Annals of the Rheumatic Diseases, vol. 67, no. 6, pp. 741-749, 2008.

[93] J. A. Pérez-Simón, S. Tabera, M. E. Sarasquete et al., "Mesenchymal stem cells are functionally abnormal in patients with immune thrombocytopenic purpura," Cytotherapy, vol. 11, no. 6, pp. 698-705, 2009.

[94] Y. Nie, C. Lau, A. Lie, G. Chan, and M. Mok, "Defective phenotype of mesenchymal stem cells in patients with systemic lupus erythematosus," Lupus, vol. 19, no. 7, pp. 850-859, 2010.

[95] L. Y. Sun, H. Y. Zhang, X. B. Feng, Y. Y. Hou, L. W. Lu, and L. M. Fan, "Abnormality of bone marrow-derived mesenchymal stem cells in patients with systemic lupus erythematosus," Lupus, vol. 16, no. 2, pp. 121-128, 2007.

[96] P. Cipriani, S. Guiducci, I. Miniati et al., "Impairment of endothelial cell differentiation from bone marrow-derived mesenchymal stem cells: new insight into the pathogenesis of systemic sclerosis," Arthritis and Rheumatism, vol. 56, no. 6, pp. 1994-2004, 2007.

[97] L. Shin and D. A. Peterson, "Impaired therapeutic capacity of autologous stem cells in a model of type 2 diabetes," Stem Cells Translational Medicine, vol. 1, no. 2, pp. 125-135, 2012.

[98] S. Kume, S. Kato, S.-I. Yamagishi et al., "Advanced glycation endproducts attenuate human mesenchymal stem cells and prevent cognate differentiation into adipose tissue, cartilage, and bone," Journal of Bone and Mineral Research, vol. 20, no. 9, pp. 16471658, 2005.

[99] C. Cramer, E. Freisinger, R. K. Jones et al., "Persistent high glucose concentrations alter the regenerative potential of mesenchymal stem cells," Stem Cells and Development, vol. 19, no. 12, pp. 1875-1884, 2010.

[100] L. Acosta, A. Hmadcha, N. Escacena et al., "Adipose mesenchymal stromal cells isolated from type 2 diabetic patients display reduced fibrinolytic activity," Diabetes, vol. 62 , no. 12 , pp. $4266-$ 4269, 2013.

[101] B. Wang, S.-M. Wu, T. Wang et al., "Pre-treatment with bone marrow-derived mesenchymal stem cells inhibits systemic intravascular coagulation and attenuates organ dysfunction in lipopolysaccharide-induced disseminated intravascular coagulation rat model," Chinese Medical Journal, vol. 125, no. 10, pp. 1753-1759, 2012.

[102] G. Moll, I. Rasmusson-Duprez, L. von Bahr et al., "Are therapeutic human mesenchymal stromal cells compatible with human blood?" Stem Cells, vol. 30, no. 7, pp. 1565-1574, 2012.
[103] S. Benvenuti, I. Cellai, P. Luciani et al., "Rosiglitazone stimulates adipogenesis and decreases osteoblastogenesis in human mesenchymal stem cells," Journal of Endocrinological Investigation, vol. 30, no. 9, pp. RC26-RC30, 2007.

[104] S. Lee, J.-R. Park, M.-S. Seo et al., "Histone deacetylase inhibitors decrease proliferation potential and multilineage differentiation capability of human mesenchymal stem cells," Cell Proliferation, vol. 42, no. 6, pp. 711-720, 2009.

[105] G. Moll, A. Hult, L. von Bahr et al., "Do ABO blood group antigens hamper the therapeutic efficacy of mesenchymal stromal cells?" PLoS ONE, vol. 9, no. 1, Article ID e85040, 2014.

[106] C. A. Herberts, M. S. G. Kwa, and H. P. H. Hermsen, "Risk factors in the development of stem cell therapy," Journal of Translational Medicine, vol. 9, article 29, 2011.

[107] M. Breitbach, T. Bostani, W. Roell et al., "Potential risks of bone marrow cell transplantation into infarcted hearts," Blood, vol. 110, no. 4, pp. 1362-1369, 2007.

[108] U. Kunter, S. Rong, P. Boor et al., "Mesenchymal stem cells prevent progressive experimental renal failure but maldifferentiate into glomerular adipocytes," Journal of the American Society of Nephrology, vol. 18, no. 6, pp. 1754-1764, 2007.

[109] Y. Wang, D. I. Huso, J. Harrington et al., "Outgrowth of a transformed cell population derived from normal human BM mesenchymal stem cell culture," Cytotherapy, vol. 7, no. 6, pp. 509-519, 2005.

[110] G. V. Røsland, A. Svendsen, A. Torsvik et al., "Long-term cultures of bone marrow-derived human mesenchymal stem cells frequently undergo spontaneous malignant transformation," Cancer Research, vol. 69, no. 13, pp. 5331-5339, 2009.

[111] L. Sensebé, M. Gadelorge, and S. Fleury-Cappellesso, "Production of mesenchymal stromal/stem cells according to good manufacturing practices: a review," Stem Cell Research and Therapy, vol. 4, no. 3, article 66, 2013.

[112] P. Gálvez, B. Clares, M. Bermejo, A. Hmadcha, and B. Soria, "Standard requirement of a microbiological quality control program for the manufacture of human mesenchymal stem cells for clinical use," Stem Cells and Development, vol. 23, no. 10, pp. 1074-1083, 2014.

[113] D. G. Phinney, "Functional heterogeneity of mesenchymal stem cells: implications for cell therapy," Journal of Cellular Biochemistry, vol. 113, no. 9, pp. 2806-2812, 2012.

[114] D. G. Phinney, G. Kopen, W. Righter, S. Webster, N. Tremain, and D. J. Prockop, "Donor variation in the growth properties and osteogenic potential of human marrow stromal cells," Journal of Cellular Biochemistry, vol. 75, no. 3, pp. 424-436, 1999.

[115] J. Ancans, "Cell therapy medicinal product regulatory framework in Europe and its application for MSC-based therapy development," Frontiers in Immunology, vol. 3, article 253, 2012. 

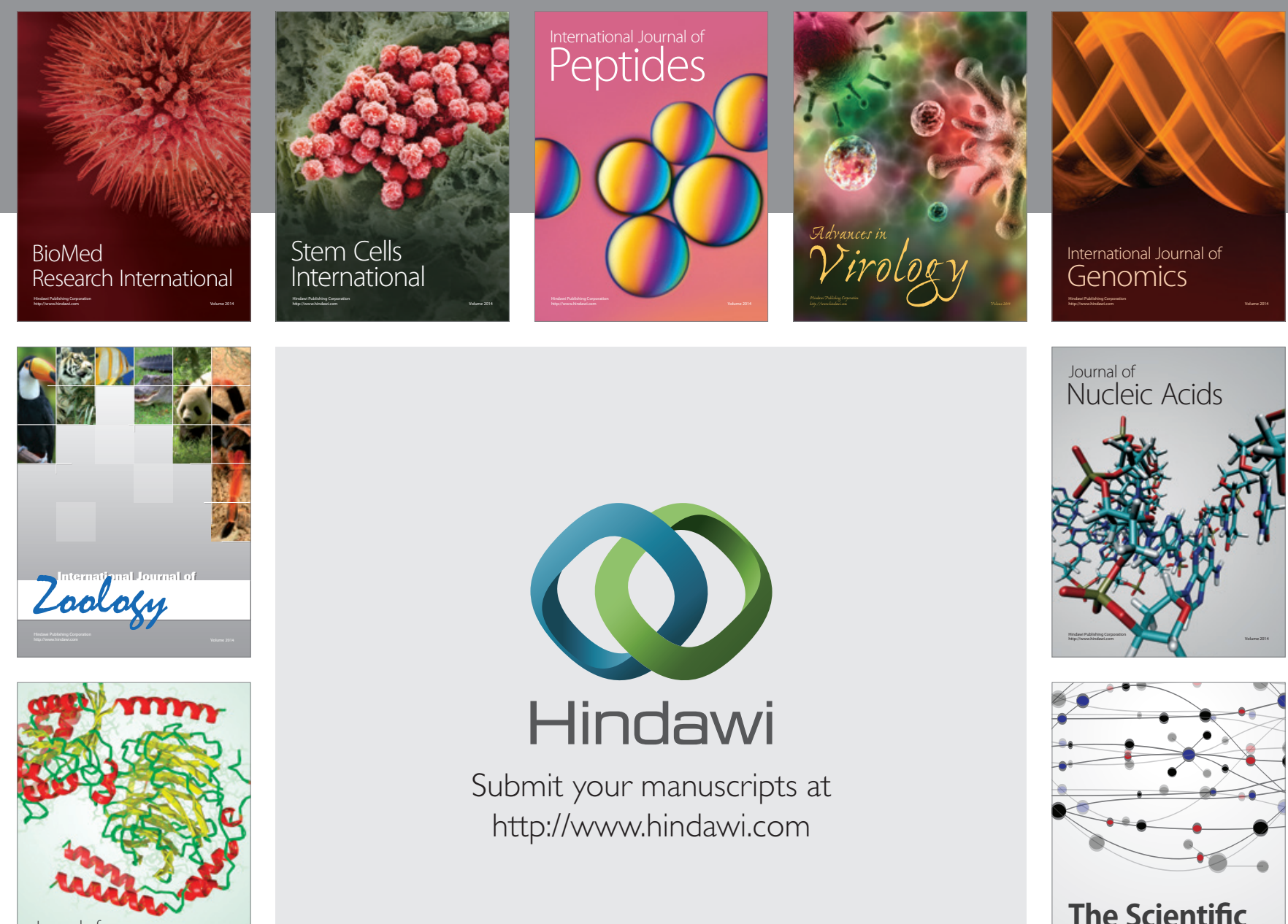

Submit your manuscripts at

http://www.hindawi.com

Journal of
Signal Transduction
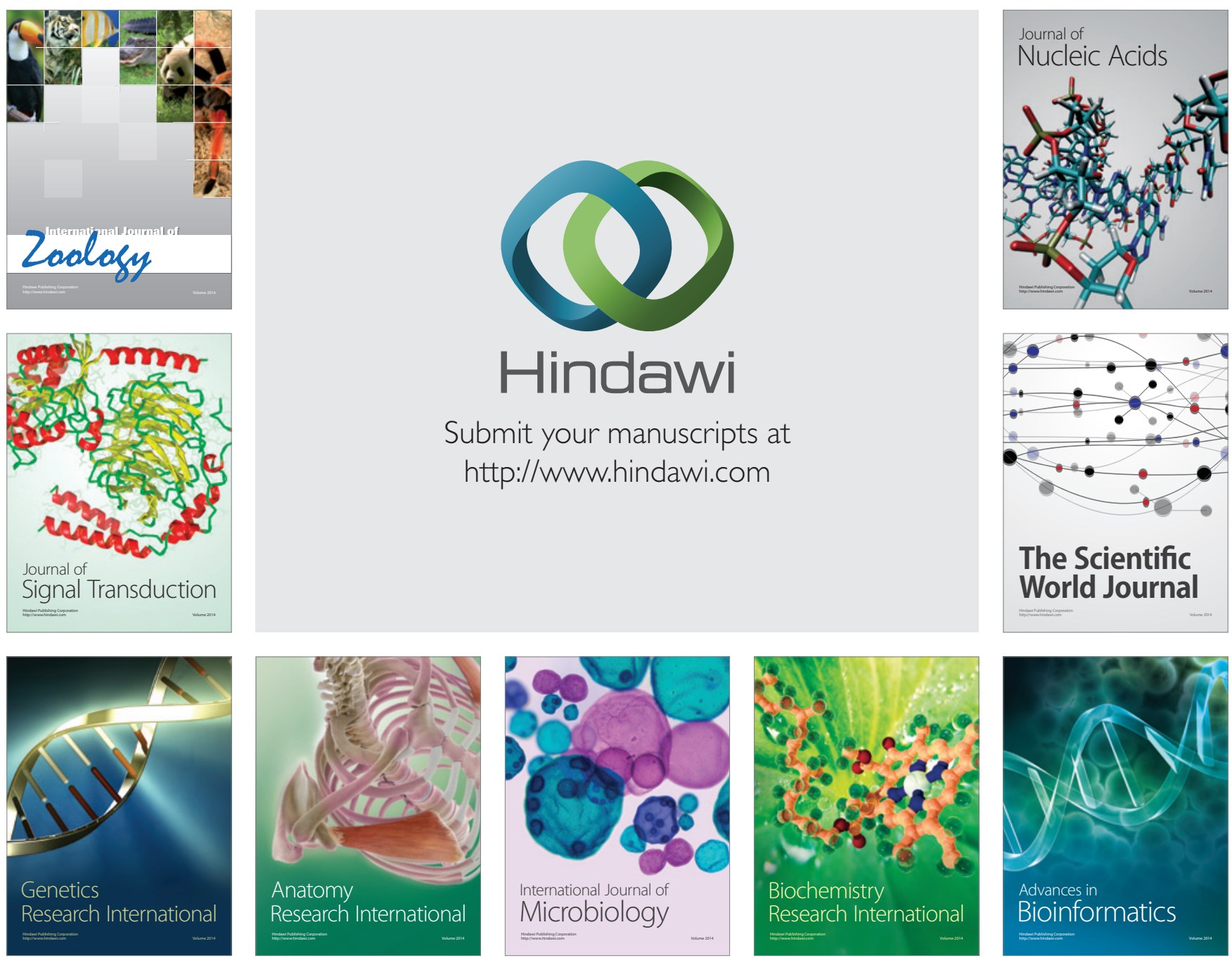

The Scientific World Journal
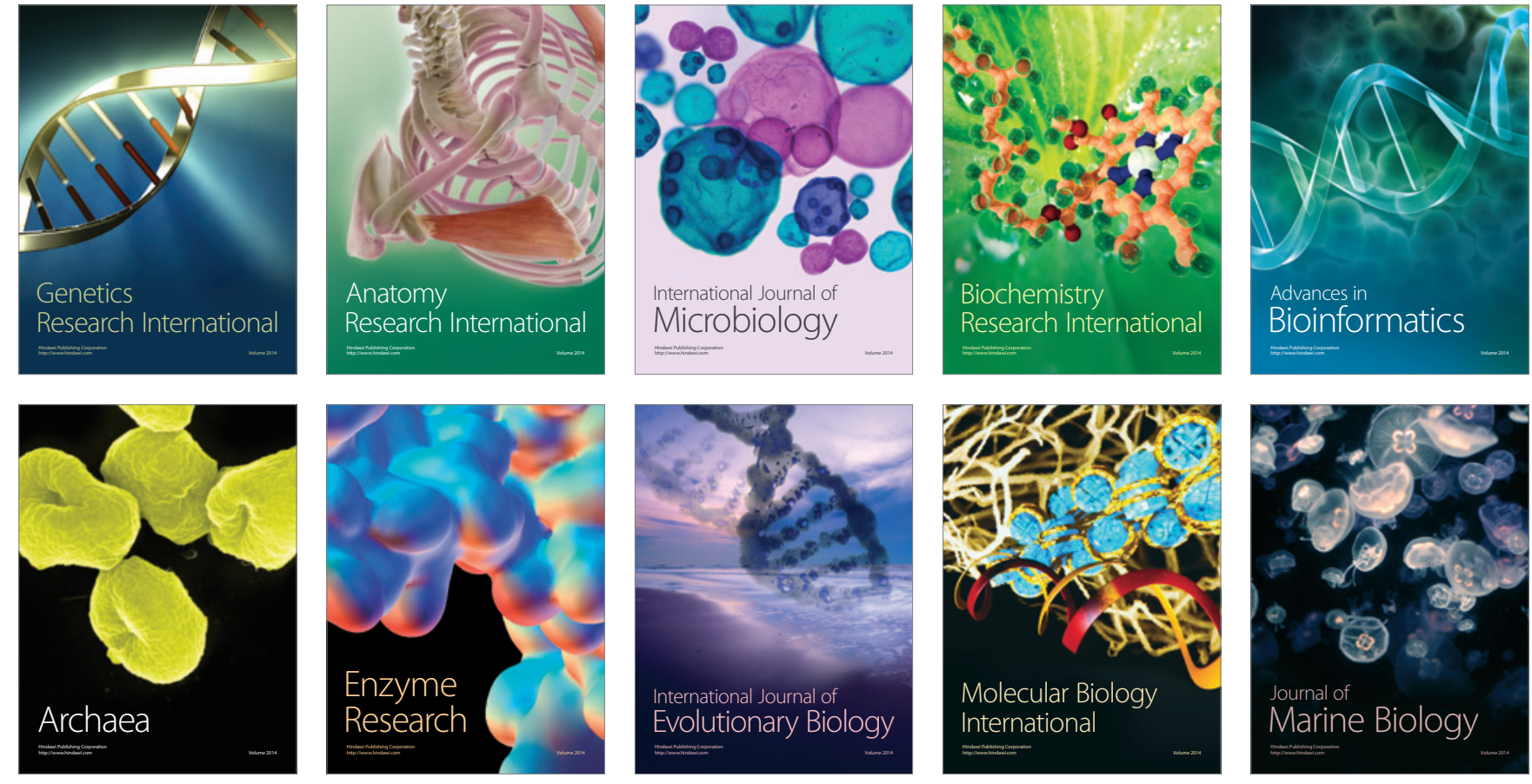